\title{
Correction to: Serologic evidence of West Nile virus and Saint Louis encephalitis virus in horses from Southern Brazil
}

\author{
Matheus N. Weber ${ }^{1}$ - Ana C. S. Mosena ${ }^{2} \cdot$ Letícia F. Baumbach $^{2} \cdot$ Mariana S. da Silva $^{2} \cdot$ Raíssa Canova $^{2}$. \\ Débora R. L. dos Santos ${ }^{3} \cdot$ Renata da F. Budaszewski ${ }^{2} \cdot$ Livia V. de Oliveira ${ }^{4} \cdot$ Michel M. Soane $^{5} \cdot$ Natália B. Saraiva ${ }^{5}$. \\ Fernanda T. Bellucco ${ }^{5}$. Bruno Amaral Mazurek ${ }^{5}$. Gustavo N. Diehl ${ }^{6}$. Laura H. V. G. Gil ${ }^{4}$. Mauro R. Borba ${ }^{7}$ \\ Luis G. Corbellini ${ }^{7}$. Cláudio W. Canal ${ }^{2}$
}

Published online: 28 April 2021

(c) Sociedade Brasileira de Microbiologia 2021

\section{Correction to: Brazilian Journal of Microbiology https://doi.org/10.1007/s42770-021-00474-7}

In the original publication, the twelfth author was missing. The author name and his affiliation are as follows.

Bruno Amaral Mazurek

EUROIMMUN Brazil, São Caetano do Sul, SP, Brazil

Publisher's note Springer Nature remains neutral with regard to jurisdictional claims in published maps and institutional affiliations.

The original article can be found online at https://doi.org/10.1007/ s42770-021-00474-7

Matheus N. Weber

matheusnweber@gmail.com

1 Laboratório de Microbiologia Molecular, Instituto de Ciências da Saúde, Universidade Feevale, Novo Hamburgo, RS, Brazil

2 Laboratório de Virologia, Faculdade de Veterinária, Universidade Federal do Rio Grande do Sul (UFRGS), Porto Alegre, RS, Brazil

3 Instituto de Veterinária, Universidade Federal Rural do Rio deJaneiro (UFRRJ), Seropédica, RJ, Brazil

4 Instituto Ageu Magalhães, Fundação Oswaldo Cruz (FIOCRUZ), Recife, PE, Brazil

5 EUROIMMUN Brazil, São Caetano do Sul, SP, Brazil

6 Secretaria da Agricultura, Pecuária e Desenvolvimento Rural do Rio Grande do Sul (SEAPDR-RS), Porto Alegre, RS, Brazil

7 Laboratório de Epidemiologia Veterinária, Faculdade de Veterinária, Universidade Federal do Rio Grande do Sul (UFRGS), Porto Alegre, RS, Brazil 